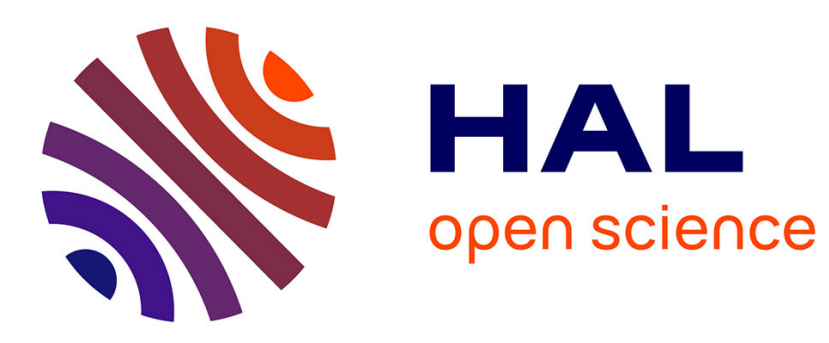

\title{
Automatic inferior vena cava segmentation in contrast-enhanced CT volumes
}

Thierry Lefevre, Benoît Mory, Roberto Ardon, Javier Sanchez-Castro, Anthony Yezzi

\section{- To cite this version:}

Thierry Lefevre, Benoît Mory, Roberto Ardon, Javier Sanchez-Castro, Anthony Yezzi. Automatic inferior vena cava segmentation in contrast-enhanced CT volumes. 2010 IEEE International Symposium on Biomedical Imaging: From Nano to Macro, Apr 2010, Rotterdam, Netherlands. pp.420-423, 10.1109/ISBI.2010.5490321 . hal-00508788

\section{HAL Id: hal-00508788 \\ https://hal.science/hal-00508788}

Submitted on 5 Aug 2010

HAL is a multi-disciplinary open access archive for the deposit and dissemination of scientific research documents, whether they are published or not. The documents may come from teaching and research institutions in France or abroad, or from public or private research centers.
L'archive ouverte pluridisciplinaire HAL, est destinée au dépôt et à la diffusion de documents scientifiques de niveau recherche, publiés ou non, émanant des établissements d'enseignement et de recherche français ou étrangers, des laboratoires publics ou privés. 


\section{AUTOMATIC INFERIOR VENA CAVA SEGMENTATION IN CONTRAST-ENHANCED CT VOLUMES}

\author{
Thierry Lefevre, Benoit Mory, \\ Roberto Ardon, Javier Sanchez-Castro
}

Medisys Research Lab, Philips Healthcare, France

\author{
Anthony Yezzi \\ Georgia Institute Of Technology, United States
}

\begin{abstract}
This paper presents a novel robust automatic method for the segmentation of the Inferior Vena Cava (IVC) in the proximity of the liver. In clinical diagnosis and surgery planning, IVC segmentation is essential since it strongly impacts both liver volumetry accuracy and vascularity analysis. Given the anatomical variability, the lack of clear boundaries and complexity of the surrounding structures along the IVC, its segmentation remains a difficult and open problem. To cope with such challenging conditions, we developed an implicit representation of a generalized cylinder and optimized a local region-based criterion under dedicated anatomical constraints. Our method was tested on a dataset of 20 contrastenhanced CT scans, achieving $80 \%$ success rate in fully automatic mode. The remaining cases needed minimal user input (one point) to reach $95 \%$ success under radiology expert criteria.
\end{abstract}

\section{INTRODUCTION}

The Inferior Vena Cava (IVC) is the large vein that carries de-oxygenated blood from the lower half of the body into the right atrium of the heart. Before reaching the heart, the IVC passes through the liver without irrigating it. In the context of liver clinical diagnosis, pre-operative planning and therapy, IVC segmentation from CT scans plays a crucial role. This role is twofold. First, the accuracy of liver volumetry is critical for liver donor transplantation, and considering the IVC as part of the liver significantly overestimates the volume of its parenchyma. Second, the IVC is an essential landmark for the segmentation of the hepatic and portal vascular trees (brightest vessels in Fig.1) which are the base for the definition of liver segments, following a Couinaud anatomical model [1].

In clinical practice, manual segmentation by radiology experts is time-consuming, subjective and difficult. Recently, several fully automatic algorithms for liver segmentation have been proposed and validated against manual segmentation references [2]. However, only few attempts to automatically exclude the IVC from the liver segmentation have been carried out [3, 4]. Automatic IVC segmentation remains difficult given the anatomical inter-patient variability, the lack of welldefined boundaries with the liver parenchyma, the possible presence of nearby tumors and the effect of non-uniform distribution of the contrast agent. Consequently, existing approaches weakly perform under non-ideal scenarios, and the problem remains unresolved up to now. To cope with the aforementioned difficulties, our contribution is twofold: (1) a model of the IVC as an implicit representation of a generalized cylinder and (2) a local region-based optimization criterion under dedicated anatomical constraints.

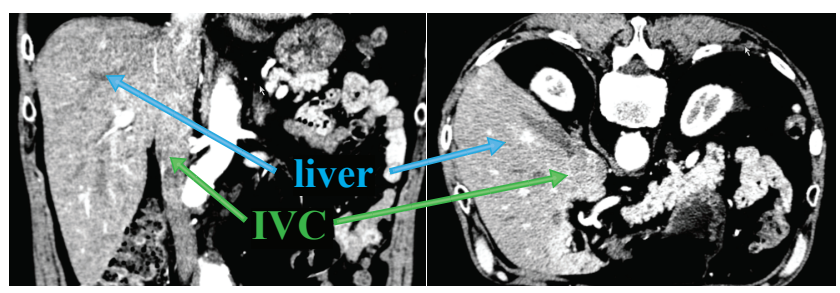

Fig. 1. Coronal/axial views of a contrast-enhanced liver CT scan, showing lack of clear boundaries between the IVC and liver parenchyma.

Vascular analysis has been a very active field of research in image processing. In particular, scale-space theory, medial representations [5], and propagation methods have helped the development of now well-established algorithms for vessel enhancement [6] and centerline extraction [7]. From our experiments, such multi-scale analysis of ridge-like curvilinear structures were insufficient to obtain an accurate delineation of the IVC lumen due to the complexity of the surrounding structures and the lack of clear boundaries in large portions of the IVC surface. Variational segmentation methods, such as implicit surface evolution schemes, minimizing boundarybased [8] or more robust region-based [9] criteria, have also been proposed as a complement to former approaches. Recently, explicit tubular representations were used $[10,11]$ in a similar context. Related to these works, we propose a variational approach where optimization is done over an implicit generalized cylinder representation with convolution surfaces. These geometrical models, originally developed in computer graphics modeling and animation [12], were recently used for visualization of complex branching vessels [13]. A key novelty of our work is to incorporate a vessel model with convolution surfaces into a region-based varia- 
tional segmentation framework (section 2). For the specific application of IVC segmentation, we increase this model robustness with dedicated constraints deduced from other anatomical structures (section 3 ) to provide clinical practitioners with a fully automatic tool.

\section{MODEL DESCRIPTION}

We formalize IVC segmentation as an optimal two-phase separation problem, in which foreground and background correspond to the IVC lumen and the surrounding structures (e.g. liver, aorta, intestines), respectively. More specifically, our model relies on an implicit tubular representation of the foreground region.

Many formulations of two-phase image segmentation consider the optimal partition as the best trade-off between shape regularity and region homogeneity. Over the set of all possible partitions $\{\mathcal{A}\}$ of a domain $\Omega$, an objective criterion is defined as the sum of some regularization term $\mathcal{R}(\mathcal{A})$ and image-dependent homogeneity measures $r_{1}$ and $r_{2}$ integrated over the foreground and background regions:

$$
\min _{\mathcal{A}}\left\{\mathcal{R}(\mathcal{A})+\int_{\mathcal{A}} r_{1}(\mathbf{x}) d \mathbf{x}+\int_{\Omega \backslash \mathcal{A}} r_{2}(\mathbf{x}) d \mathbf{x}\right\}
$$

With known intensity distributions $p_{i}$ for each region, a common choice is to set $r_{i}(\mathbf{x})=-\log p_{i}(I(\mathbf{x}))$ [14]. In a continuous setting, an implicit representation of $\mathcal{A}$ can be used, defining a real function $\Phi$, positive in the foreground. Using the Heaviside function $H, H(\Phi)$ is the characteristic function of the foreground region $\mathcal{A}$, and (1) is equivalent to

$$
\begin{gathered}
\left.\min _{\Phi}\left\{\mathcal{R}(\Phi)+\int_{\Omega} H(\Phi(\mathbf{x})) r(\mathbf{x})\right) d \mathbf{x}\right\} \\
\text { with } r(\mathbf{x})=r_{1}(\mathbf{x})-r_{2}(\mathbf{x})
\end{gathered}
$$

Assuming a circular cross-section, a vessel branch is often modeled by an open parameterized medial curve $\mathbf{m}(s)$ : $[0,1] \rightarrow \mathbb{R}^{3}$, with a set of corresponding point-wise radii. The key idea of our model is to define an implicit analytical representation of such a generalized cylinder. This representation is obtained by integrating a smooth decreasing radial function $\omega$ centered all over the medial curve $\mathbf{m}(s)$, with continuouslyvarying scales $\sigma(s)$ (see Fig. 2):

$$
\Phi_{\mathbf{m}, \sigma}(\mathbf{x})=\int_{0}^{1} \omega\left(\frac{\|\mathbf{x}-\mathbf{m}(s)\|}{\sigma(s)}\right)\left\|\mathbf{m}^{\prime}(s)\right\| d s-C
$$

where $\mathbf{x}$ is any point in the domain $\Omega, C$ an arbitrary positive constant and $\omega$ is typically a Gaussian function. The term $\left\|\mathbf{m}^{\prime}(s)\right\|$ ensures invariance with respect to parameterization.

Substituting the vessel representation (3) into the objective criterion (2) and associating specific regularization terms to the unknown centerline $\mathbf{m}(s)$ and scales $\sigma(s)$ yields:

$$
\min _{\mathbf{m}, \sigma}\left\{\mathcal{R}(\mathbf{m}, \sigma)+\int_{\Omega} H\left(\Phi_{\mathbf{m}, \sigma}(\mathbf{x})\right) r(\mathbf{x}) d \mathbf{x}\right\},
$$

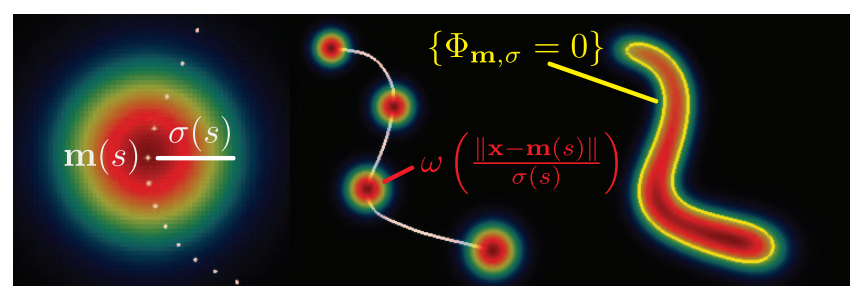

Fig. 2. Analytical implicit representation of a vessel in 2D.

where $\mathcal{R}(\mathbf{m}, \sigma)$ is designed to penalize both the length of the centerline and the variations of scale along it, controlled by the positive scalar parameters $\lambda$ and $\mu$ respectively:

$$
\mathcal{R}(\mathbf{m}, \sigma)=\lambda \int_{0}^{1}\left\|\mathbf{m}^{\prime}(s)\right\|+\mu \int_{0}^{1}\left|\sigma^{\prime}(s)\right|^{2}
$$

The Euler-Lagrange equations of the functional in (4) can be found by standard calculus of variations, made easier by the analytical expression of the implicit representation. Due to space limitations, we have omitted the details of these computations. They result in gradient-descent evolution equations for both the centerline and the scales, of the form ${ }^{1}$ (see Fig.3):

$$
\begin{aligned}
\frac{\partial \mathbf{m}}{\partial t}(s) & =\lambda \kappa(s) \mathbf{n}(s)-\int_{\left\{\Phi_{\mathbf{m}, \sigma}=0\right\}} r(\mathbf{x}) \mathbf{N}(\mathbf{x}, s) d \mathbf{x} \\
\frac{\partial \sigma}{\partial t}(s) & =\mu \sigma^{\prime \prime}(s)-\int_{\left\{\Phi_{\mathbf{m}, \sigma}=0\right\}} r(\mathbf{x}) B(\mathbf{x}, s) d \mathbf{x}
\end{aligned}
$$

where $\kappa$ is the curvature of the centerline, $\mathbf{n}=\mathbf{m}^{\prime \prime} /\left\|\mathbf{m}^{\prime \prime}\right\|$ its normal and functions $\mathbf{N}(\mathbf{x}, s)$ and $B(\mathbf{x}, s)$ are given by

$$
\begin{aligned}
& \mathbf{N}(\mathbf{x}, s)=\frac{\omega^{\prime}\left(\frac{\|\mathbf{x}-\mathbf{m}(s)\|}{\sigma(s)}\right)}{\left\|\nabla \Phi_{\mathbf{m}, \sigma}(\mathbf{x})\right\|} \frac{\left\|\mathbf{m}^{\prime}(s)\right\|}{\sigma(s)} \frac{\mathbf{x}-\mathbf{m}(s)}{\|\mathbf{x}-\mathbf{m}(s)\|} \\
& B(\mathbf{x}, s)=-\frac{\omega^{\prime}\left(\frac{\|\mathbf{x}-\mathbf{m}(s)\|}{\sigma(s)}\right)}{\left\|\nabla \Phi_{\mathbf{m}, \sigma}(\mathbf{x})\right\|}\left\|\mathbf{m}^{\prime}(s)\right\| \frac{\|\mathbf{x}-\mathbf{m}(s)\|}{\sigma(s)^{2}}
\end{aligned}
$$

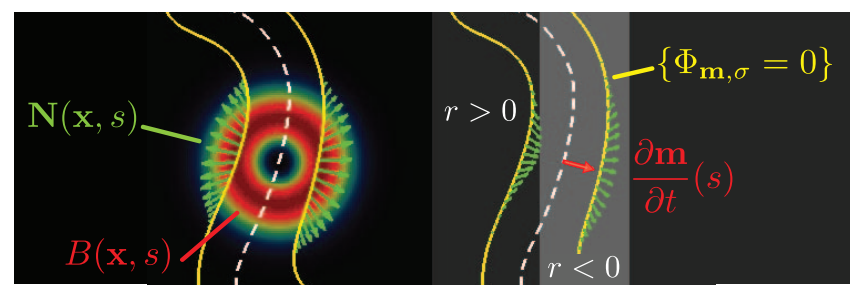

Fig. 3. Left: vector field $\mathbf{N}(\mathbf{x}, s)$ plotted only on the boundary $\left\{\Phi_{\mathbf{m}, \sigma}=0\right\}$, and $B(\mathbf{x}, s)$, color-coded. Right: Evolution of a centerline point with Eq.(6) as the sum of all forces $-r . \mathbf{N}$ computed on the boundary, for a simple image term $r(\mathbf{x})$ shown in gray.

These general evolution equations can be further adapted to IVC segmentation by designing specific homogeneity mea-

\footnotetext{
${ }^{1}$ We have here neglected terms derived from parameterization invariance.
} 
sures $r_{1}$ and $r_{2}$ that capture the local variations inside the lumen due to contrast product injection and the complex spacevarying statistics of the outside tissues. A local likelihood criterion is defined at every $\mathbf{x}$ from a weighted combination of local probability densities $p_{i}(I(\mathbf{x}), s)$ along the centerline:

$$
r_{i}(\mathbf{x})=-\int_{0}^{1} \omega\left(\frac{\|\mathbf{x}-\mathbf{m}(s)\|}{a}\right) \log p_{i}(I(\mathbf{x}), s) d s
$$

where the blending scale $a$ is related to the expected statistical intensity variations along the vessel [15]. The probability densities $p_{i}$ are non-parametric and updated periodically during optimization using kernel density estimation.

\section{AUTOMATIC IVC SEGMENTATION}

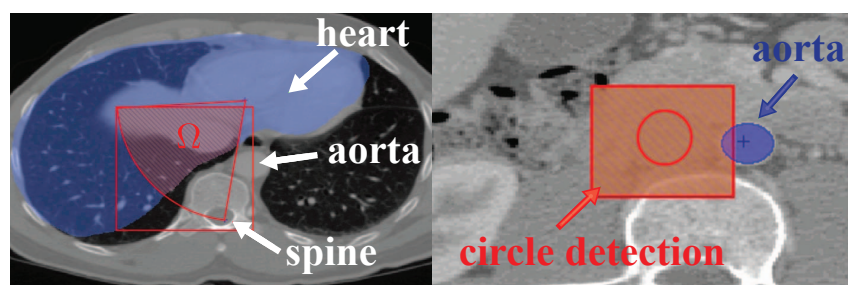

Fig. 4. Left: the hashed area is an axial view of the restriction of our computation domain $\Omega$. Right: Square represents the area where the circle detection filter is applied to locate the second IVC point.

Combined with a local region-based criterion, the previous implicit vessel model has the required ability to follow image evidence where available and naturally extrapolate the surface where it is missing. Nevertheless, fully automatic and robust segmentation of the IVC requires significant additional effort. As a non-convex functional, (4) has obviously multiple local minima, and the IVC is only expected to be one among those. Therefore, special attention should be paid to the domain $\Omega$, fixed during the optimization, and the initial state $\left(\mathbf{m}_{0}, \sigma_{0}\right)$ of the tubular model evolution.

Dedicated anatomical constraints: The IVC enters the heart from its right lower side, runs near the liver, along the vertebral column. Incorporating additional knowledge on these anatomical structures (heart, liver, spine) allows to restrict the computation domain $\Omega$ and avoid possible mistakes. To do so, their segmentations need not be precise. Localization of the vertebral column is obtained by a simple thresholding since bone intensity values (Hounsfield Units) are generally above ( $>500 \mathrm{HU}$ ) those of soft tissues $(<200 \mathrm{HU})$. Similarly, lungs are easily detected (air is $-1000 \mathrm{HU}$ ) and an approximate positioning of the heart can then be deduced. The more challenging task of liver segmentation has been extensively studied in recent years, hence we assume it is available. The last anatomical entity that we detect is the aorta. Unlike the IVC, this artery, which also runs along the back bone, is highly visible. Its segmentation is generated using the model presented in the previous section, taking as an initialization a line between two initial points: one in the heart and the other found inside the aorta from the spine position. Finally, the computation domain $\Omega$ is defined as a $3 \mathrm{D}$ extrusion along the spine axis of an angular sector positioned using all recovered anatomical information (see Fig.4 left).

Model initialization: The initialization of gradient-descent equations (6) influences the outcome of our algorithm as well as its convergence speed. We position the curve $\mathbf{m}_{0}$ by computing a minimal path within our restricted computation domain. The minimal path joins two points, the first located inside the heart and the second in a region underneath the liver. This second point is found as the best response of a standard 2D morphological filter designed for circle detection, applied on the last axial slice intersecting the liver and in a neighborhood of the previously segmented aorta (see Fig. 4 right). The minimal path is computed using Dijkstra's algorithm, penalizing the square difference to the image intensity at the extremity points. More precisely, if $I_{1}, I_{2}$ are the image intensities at at the two extremities, penalization is $\mathcal{P}(\mathbf{x})=\left(I(\mathbf{x})-\frac{I_{1}+I_{2}}{2}\right)^{2}$. The initial scale $\sigma_{0}$ is simply set to a constant, deduced from an anatomical mean radius of $12.5 \mathrm{~mm}$.

\section{RESULTS AND CONCLUSION}

The automatic IVC segmentation algorithm has been applied to a database of 20 contrast-enhanced CT volumes acquired in portal venous phase from scanners of different manufacturers with a pixel size ranging from 0.63 to $0.78 \mathrm{~mm}$ and inter-slice distance of $2 \mathrm{~mm}$. 16 cases were considered by an expert radiologist as satisfactory, while 3 cases needed a minimal user interaction in the initialization step. Two fully automatic results are shown in Fig.5. In one single case, the surface evolution was unable to recover the hardly visible boundaries because of a excessively poor image quality and unusual patient anatomy.

The promising results of this preliminary evaluation validate our choice of a model-based variational approach with local region homogeneity criteria under specific anatomical constraints. The application described in this paper is already a useful clinical tool, reaching $80 \%$ success rate fullyautomatically and $95 \%$ interactively. It also constitutes a first step towards automatic Couinaud liver segments extraction.

To the best of our knowledge, this is also the first use of implicit convolution surfaces for image segmentation. In general, the advantage of this hybrid representation is to model smooth complex objects with only simple explicit geometrical primitives, here a single curve. In the particular context of variational image segmentation, the original aspect is to induce the motion of these simple primitives from the analysis of the implicitly-generated surface. Future extensions to smooth branching structures, vascular trees and other medial representations are exciting perspectives. 


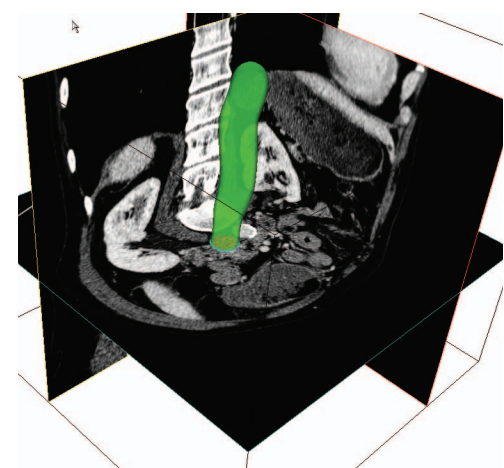

(a)

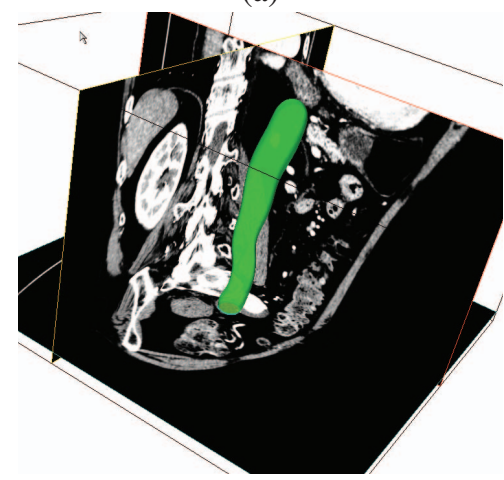

(b)

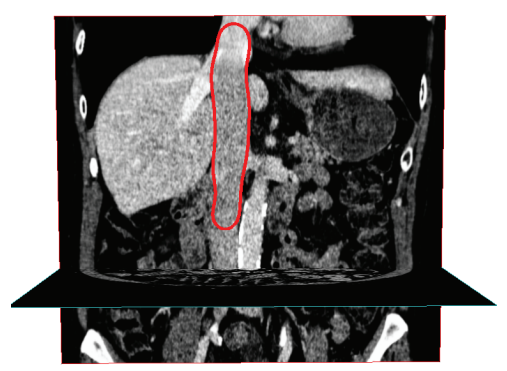

(c)

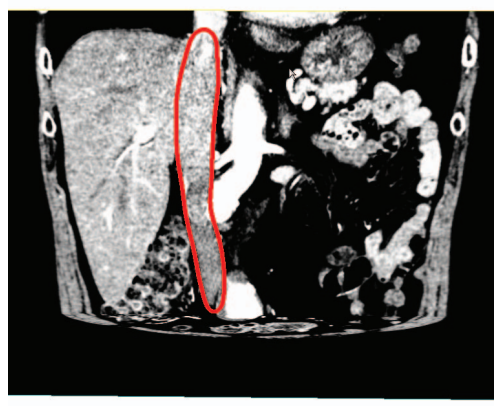

(d)

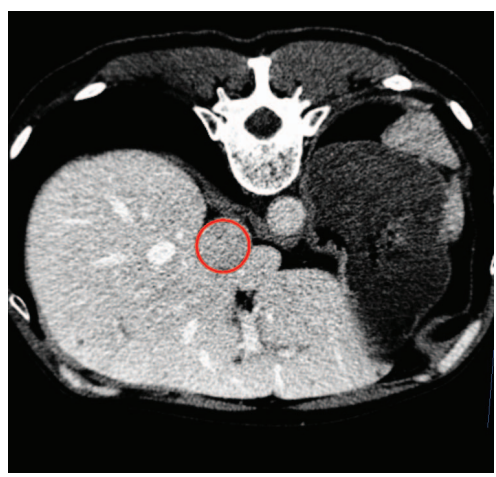

(e)

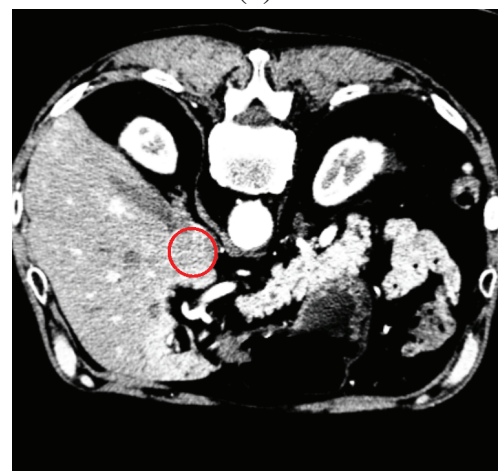

(f)

Fig. 5. Segmentation of the IVC in two different CT volumes, in each row. (a)-(b) show a 3D surface rendering, (c)-(d) sagittal views and (e)/(f) axial views, showing the algorithm performance in the absence of clear boundaries with the liver parenchyma.

\section{REFERENCES}

[1] A.Mihalcea et. al., "A new pc based software for semi automatic liver segmentation. clinical study for preoperative tumor localization," InfoRAD 2003, Radiological Society of North America RSNA, 2003.

[2] T. Heimann, B. van Ginneken, and Eds. M. Styner, "3D segmentation in the clinic: A grand challenge," MICCAI, 2007.

[3] L. Rusko, G. Bekes, G. Nemeth, and M. Fidrich, "Fully automatic liver segmentation for contrast-enhanced CT images," MICCAI, 3D Segmentation in the Clinic: A Grand Challenge, pp. 143-150, 2007.

[4] Y.Chi et. al., "A discussion on the evaluation of a new automatic liver volume segmentation method for specified CT image datasets," MICCAI, pp. 167-175, 2007.

[5] S.M. Pizer, D. Eberly, B.M. Morse, and D.S. Fritsch, "Zoominvariant vision of figural shape: The mathematics of cores," Computer Vision and Image Understanding, 1996.

[6] A.F. Frangi, W.J. Niessen, R.M. Hoogeveen, T. van Walsum, and M.A. Viergever, "Model-based quantification of 3d magnetic resonance angiographic images," IEEE TMI, vol. 18, no. 10, pp. 946-956, 1999.

[7] S. Aylward and E. Bullitt, "Initialization, noise, singularities, and scale in height ridge traversal for tubular object centerline extraction," IEEE TMI, vol. 21, no. 2, pp. 61-75, 2002.
[8] R. Manniesing et. al., "Level set based cerebral vasculature segmentation and diameter quantification in CT angiography," Medical Image Analysis, vol. 10, pp. 200-214, 2006.

[9] M. Hernandez and A.F. Frangi, "Non-parametric geodesic active regions: Method and evaluation for cerebral aneurysms segmentation in 3DRA and CTA," Medical Image Analysis, vol. 11, no. 3, pp. 224-241, 2007.

[10] H.Li and A. J. Yezzi, "Vessels as 4-d curves: Global minimal 4-d paths to extract 3-d tubular surfaces and centerlines," IEEE TMI, vol. 26, no. 9, pp. 1213-1223, 2007.

[11] Julien Mille, Romuald Boné, and Laurent D. Cohen, "Regionbased $2 \mathrm{~d}$ deformable generalized cylinder for narrow structures segmentation," in ECCV (2), 2008, pp. 392-404.

[12] Jules Bloomenthal and Ken Shoemake, "Convolution surfaces," Computer Graphics, vol. 25, no. 4, pp. 251-256, July 1991, Proceedings of SIGGRAPH'91.

[13] S. Oeltze and B. Preim, "Visualization of vasculature with convolution surfaces: Method, validation and evaluation," IEEE TMI, vol. 24, no. 4, pp. 540-548, Apr. 2005.

[14] S. C. Zhu and A. Yuille, "Region competition: Unifying snakes, region growing, and bayes/mdl for multiband image segmentation," IEEE Trans. On PAMI, 1996.

[15] B. Mory, R. Ardon, and Thiran J.P., "Variational segmentation using fuzzy region competition and local non-parametric probability density functions," in ICCV, Proceedings, 2007. 